\title{
Zinc(II) ion promotes anti-inflammatory effects of rhSOD3 by increasing cellular association
}

\author{
Younghwa Kim ${ }^{1, \#}$, Yoon-Jae Jeon ${ }^{2, \#}$, Kang Ryu ${ }^{3} \mathcal{E}$ Tae-Yoon Kim ${ }^{2, *}$ \\ ${ }^{1}$ Department of Emergency Medical Technology, Kyungil University, Gyeongsan 38428, ${ }^{2}$ Laboratory of Dermato-Immunology, Catholic \\ Research Institute of Medical Science, College of Medicine, The Catholic University of Korea, Seoul 06591, ${ }^{3}$ RNS BIO, Seoul 04784, Korea
}

Recently, we demonstrated that superoxide dismutase 3 (SOD3) is a strong candidate for biomedicine. Anti-oxidant function of SOD3 was accomplished without cell penetration, and it inhibited the inflammatory responses via non-enzymatic functions. SOD3 has the heparin binding domain associating cell surface. Interestingly, we found that $\mathrm{Zn}^{2+}$ promotes transduction effects of recombinant human SOD3 (rhSOD3) by increasing uptake via the heparin binding domain (HBD). We demonstrated an uptake of rhSOD3 from media to cell lysate via HBD, resulting in an accumulation of rhSOD3 in the nucleus, which was promoted by the presence of $\mathrm{Zn}^{2+}$. This resulted in increased inhibitory effects of rhSOD3 on NF-kB and STAT3 signals in the presence of $\mathrm{Zn}^{2+}$, which shows elevated association of rhSOD3 into the cells. These results suggest that an optimized procedure can help to enhance the inflammatory efficacy of rhSOD3, as a novel biomedicine. [BMB Reports 2017; 50(2): 85-90]

\section{INTRODUCTION}

Superoxide dismutases (SODs) are a group of anti-oxidant enzymes of which functions are removing reactive oxygen species (ROSs) from the cellular context and preserving cells from environmental stresses. SODs function to dismutate two superoxide radicals into a hydrogen peroxide and an oxygen molecule. Recently, Serra et al. reported that SOD3 inhibits telomere shortening, a cellular aging process, by extending the telomeric clock of human fibroblast (1). In the other hand, over-expression of SOD3 suppresses the growth of breast carcinoma and melanoma cells efficiently by the adenoviral

${ }^{*}$ Corresponding author. Tel: +82-2-2258-6221; Fax: +82-2-34828261; E-mail: tykimder@catholic.ac.kr

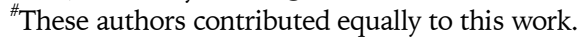

https://doi.org/10.5483/BMBRep.2017.50.2.150

Received 25 August 2016, Revised 23 September 2016, Accepted 18 November 2016

Keywords: Anti-inflammatory, Cytopermeability, Heparin Binding Domain, Superoxide dismutase 3, Zinc (II) ion transduction $(2,3)$. It has been also shown that gene therapy using SOD3 gene alleviates aorta restenosis, and mitigates collagen-induced arthritis in rodents (4). The presence of SOD3 in serum and even in extracellular matrices (ECM) plays an important role for the systemic defense mechanisms (5). Inflammatory cytokines, like IL-4, IL-1 $\alpha$ and IFN- $\gamma$ up-regulate the level of expression of SOD3, but, TNF- $\alpha$ and TGF- $\beta$ down-regulates SOD3 in vascular smooth muscle cells and human skin fibroblasts $(6,7)$. However, the underlying specific mechanisms remain to be exploited, particularly in regards with IFN- $\gamma$-induced SOD3 expression.

SODs can be further classified as: SOD1, containing $\mathrm{Cu}$ and $\mathrm{Zn}$ atoms; SOD2, containing $\mathrm{Mn}$ atom; and SOD3, located in the extracellular fluid or on the cell surface. It is important to note that, as like SOD1, SOD3 contains $\mathrm{Cu}$ and $\mathrm{Zn}$ atoms, however, a heparin-binding domain (HBD) has been characterized to exist in the C-terminal end, unlike SOD1. It has been reported that the HBD of SOD3 acts as a nuclear localization signal, and which protects genomic DNA from oxidative stress and regulates the DNA transcription which is sensitive to the detox reaction within the nuclei of testis and thymus cells (8).

Certain proteins enter cells through the plasma membranes, effectively. Several lines of studies are now actively performed to utilize such proteins to transduce with beneficial substances into cytoplasm. Typical examples include VP22 protein, HIV Tat protein, PEP-1 peptide, and ANTP (9). It has been shown that the cell-transduction property of proteins is the function of protein transduction domain (PTD), capable of crossing phospholipid bilayers of plasma membrane (10). The HBD of SOD3 has been shown to have similar properties as the other cellpenetrating peptides having highly positive charged residues.

Here, we compared rhSOD3 and 209E; a proteolytic variant of SOD3 with a missing HBD at the C-terminal end, to investigate the role of HBD in the cellular uptake. We found that $\mathrm{Zn}^{2+}$, which is one of the major cofactors of SOD3, promoted the cellular uptake and anti-inflammatory effects of rhSOD3. Therefore, we expect further optimization processes could help developing rhSOD3 and its variants as novel biomedicines. 


\section{RESULTS}

\section{Effect of cellular uptake efficiency by metal $2+$ ions in $\mathrm{HaCaT}$ and 293T cells}

Since SOD3 shows its anti-inflammatory effects by scavenging extra cellular superoxide molecules, we elevated the enzymatic activity of SOD by adding its catalytic cofactors, zinc and copper ions. To examine the potential effect of metal $2+$ ions, $\mathrm{HaCaT}$ or 293T cells were exposed to recombinant human SOD3 (rhSOD3), and incubated for $1 \mathrm{~h}$ in the presence of $\mathrm{CuSO}_{4}$ or $\mathrm{ZnCl}_{2}$. Interestingly, when $\mathrm{ZnCl}_{2}$ was added to the $\mathrm{HaCaT}$ or 293T cells, the residual rhSOD3 in the media was decreased, while rhSOD3 associated to the cell increased in a dose-dependent manner (Fig. 1A, left), indicating that zinc ions increase the cellular association or uptake of rhSOD3. However, rhSOD3 levels in media and cells remained unchanged on addition of $\mathrm{CuSO}_{4}$. Under similar conditions, rh209E, which is missing the HBD, remained unchanged in the cell lysates of both HaCaT and 293T cells (Fig. 1B, lower, right), indicating that increase of cellular association or uptake of rhSOD3 by zinc ions is mediated by HBD.

\section{Localization of exogenous rhSOD3 in the HaCaT}

In order to investigate the transduction effect of zinc ions between HBD and cell line, we studied the internalization of exogenously added fusion proteins using $\mathrm{HaCaT}$, which expresses cell surface GAGs such as heparin sulfates. Using fluorescence microscopy, we identified surface-bound and internalized rhSOD3 in HaCaT. The punctate was a typical membrane signal by fluorescence microscopy. rhSOD3 were found to be internalized, and strikingly increased the internalization of SOD3 in the presence of $\mathrm{Zn}^{2+}$ (Fig. 2A, middle lane). These results suggest that $\mathrm{Zn}^{2+}$ enhance the internalization of rhSOD3. However, rh209E, which is deficient of HBD, failed to internalize in the presence or absence of $\mathrm{Zn}^{2+}$ (Fig. 2A, right lane), supporting that HBD is critical for cellular internalization. Also, rh209E could be not internalized in the cell line lacking the ability to interact with cell surface heparin sulfate
(A)

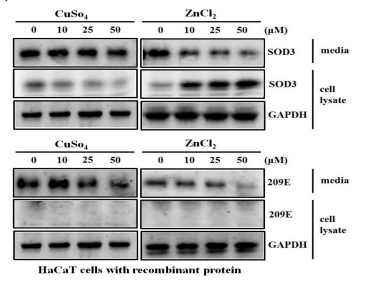

(B)

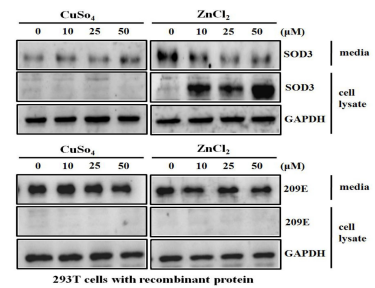

Fig. 1. $\mathrm{Zn}^{2+}$ induces uptake of rhSOD3 via heparin binding domain. HaCaT (A) and 293T cells (B) were cultured with purified rhSOD3 or rh209E at various concentrations $(0,10,25,50 \mu \mathrm{M})$ of $\mathrm{CuSO}_{4} / \mathrm{ZnCl}_{2}$. Amounts of rhSOD3 or rh209E in HaCaT and 293T cells were compared between media and cell lysates by western blot analysis. proteoglycans (HSPGs) (11), suggesting that $\mathrm{Zn}^{2+}$ plays a crucial and essential role in the uptake of rhSOD3 into the $\mathrm{HaCaT}$, which is expressed on the cell surface such as heparin sulfates.

\section{$\mathrm{Zn}^{2+}$ promotes inflammatory responses of SOD3 by} inhibiting the phosphorylation of NF-kB and STAT3 in HaCaT We first examined whether $\mathrm{Zn}^{2+}$ can promote cellular efficacy of rhSOD3 to regulate NF-kB signal in HaCaT. NF-kB is one of the important signals in the inflammatory responses. The phosphorylation of NF-kB p65 was strongly inhibited by incubation with rhSOD3 and $\mathrm{ZnCl}_{2}$, whereas $\mathrm{ZnCl} 2$ itself exhibited no inhibitory effect (Fig. 3A, 3B). When the cells were treated with $\mathrm{ZnCl}_{2}$ and rhSOD3, p-NF-kBp65 was inhibited in a dose-dependent manner (Fig. 3B). These results suggested that $\mathrm{Zn}^{2+}$ promotes the anti-inflammatory activity of SOD3 through the inhibition of the p-NF-kB65. After HaCaT were stimulated by TNF $\alpha$ and IFN $\gamma$, we investigated whether STAT3 is induced by tyrosine phosphorylation by cytokines. The pSTAT3 was strongly inhibited by treatment with rhSOD3, whereas the pSTAT3 was unaffected by rh209E (Fig. 3C- lane 1). Compared to the levels of tyrosine phosphorylation, the total levels of these proteins were not altered (Fig. 3C- lane 2). To determine the effect of $\mathrm{ZnCl}_{2}$, we analyzed mRNA expression level of IL-1 $\beta$, IL-1 $\alpha$, TNF $\alpha$, IL- 8 and IL- 6 in HaCaT. After stimulation by TNF $\alpha$ and IFN $\gamma$, the HaCaT cells were treated with rhSOD3 or rh209E, with or without $\mathrm{ZnCl}_{2}$. As

(B)
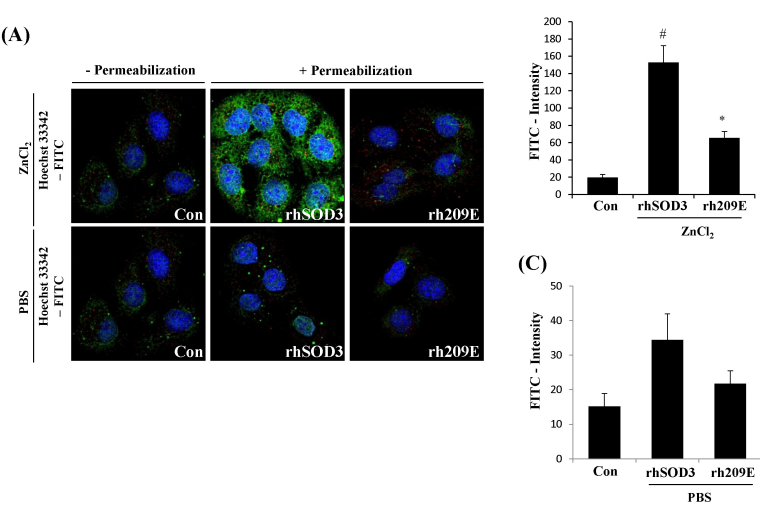

Fig. 2. $\mathrm{Zn}^{2+}$ promotes nuclear accumulation by rhSOD3, but not rh209E, in HaCaT. After incubation with/without $\mathrm{ZnCl}_{2}$, HaCaT were washed and stained with secondary antibodies labeled with FITC, prior to and after cell permeabilization, respectively. Green labeled surface-bound fraction of rhSOD3 was evident in intercellular contacts, whereas intracellular fraction of rh209E manifests that did not locate in cytosol and nuclear region. Counterstaining was labeled with nucleic acid stain, Hoechst33342 (A). The data are represented as mean \pm SEM of fluorescent intensity (B, C). Statistical comparisons between groups are made with the unpaired two-sided t-test. All experiments were performed at least three times. Differences with ${ }^{\#} \mathrm{P}<0.005$ versus Control group, and $* \mathrm{P}<0.05$ versus rhSOD3 treatment group were considered statistically significant 
(A)

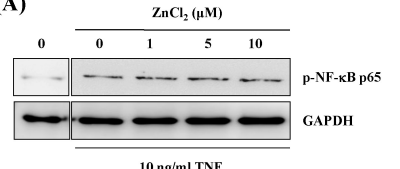

(C)

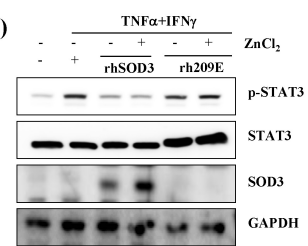

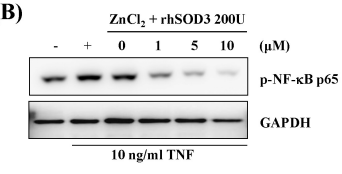

(B)
Fig. 3. Inhibition of NF-kB p65 and STAT3 signal through transduction effect rhSOD3, but not rh209E, by $\mathrm{Zn}^{2+}$ in HaCaT. The cells were treated both TNF- $\alpha(10 \mathrm{ng} / \mathrm{ml})$ and IFN $\gamma(100 \mathrm{U} / \mathrm{ml})$ and various concentration of $\mathrm{ZnCl}_{2}(0-10 \mu \mathrm{M})$ (A). Purified rhSOD3 was added to cells at $200 \mathrm{U} / \mathrm{ml}$ (B). Amounts of p-NF-kB $\mathrm{P} 65$ in the cytosol was analyzed by western blot analysis. After purified rhSOD3 and rh209E were added to cells (with/without $200 \mathrm{U} / \mathrm{mL})$, and added with/without $\mathrm{ZnCl}_{2}(10 \mu \mathrm{M})(\mathrm{C})$. All proteins were analyzed by western blot analysis.

shown in Fig. 4, in combination with rhSOD3 and $\mathrm{ZnCl}_{2}$, the levels of inflammatory cytokines are effectively reduced by $\mathrm{ZnCl}_{2}$. However, the combination of rh209E and $\mathrm{ZnCl}_{2}$ was ineffective and did not reduce the levels of inflammatory cytokines. Quantitative analysis showed that $\mathrm{Zn}^{2+}$ inhibited inflammatory responses of SOD3 caused in response to IL-1 $\beta$, IL-1 $\alpha$, TNF $\alpha$, IL-8 and IL-6 altered (Fig. 4).

\section{$\mathrm{Zn}^{2+}$ enables penetration of rhSOD3 into epidermis of mouse skin}

To evaluate effects of $\mathrm{Zn}^{2+}$, rhSOD3 is translocated to dorsal skin, sections of $30 \mu \mathrm{m}$ thickness were made with cryotome, and were then observed under confocal microscope (Supplementary 1). When applied to the skin with FITCconjugated rhSOD3 and $\mathrm{ZnCl}_{2}$, the signals of rhSOD3 were found in the epidermis. However, application of FITCconjugated rhSOD3 alone did not reveal any signals of rhSOD3 (Supplementary 1A). The same dose of FITCconjugated rhSOD3 was further analyzed and confirmed by Western blot (Supplementary 1B). We found that the presence of $\mathrm{Zn}^{2+}$ enhanced the skin permeation. This property of $\mathrm{Zn}^{2+}$ might therefore increase the penetration of rhSOD3 into viable layers of the skin, indicating that $\mathrm{Zn}^{2+}$ elevates the delivery of HBD proteins into the skin.

\section{DISCUSSION}

We have evaluated the potentiality of SOD3 as an important therapeutic agent for curing diverse diseases. However, diverse restraints prevented the product of homogenous, an active form of hSOD3 (12-14). The anti-oxidative effect of SOD3 has been well founded (15-18). Even though the role of SOD3 in
(A)

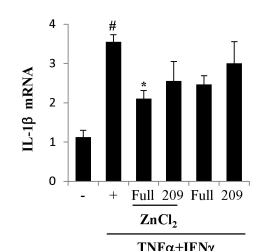

(B)
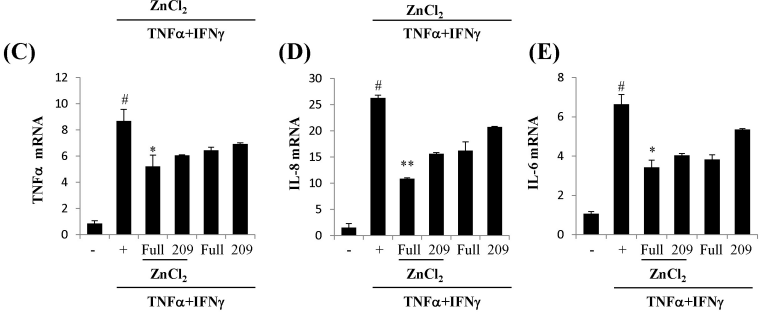

Fig. 4. $\mathrm{Zn}^{2+}$ reduces inflammatory cytokine by treating with rhSOD3 or rh209E in HaCaT. HaCaT were treated with $10 \mathrm{ng} / \mathrm{ml}$ TNF- $\alpha$ and $100 \mathrm{U} / \mathrm{ml}$ IFN- $\gamma$. Purified rhSOD3 or rh209E were added to cells at $200 \mathrm{U} / \mathrm{ml}$, and various concentration of $\mathrm{ZnCl}_{2}$ $(0-10 \mu \mathrm{M})$. Quantitative real-time PCR was performed using the KAPA SYBR fast qPCR Kit (KAPA biosystems, Woburn, MA, USA) as previously described (24). The data are represented as mean \pm SEM. Statistical comparisons between groups were made with the unpaired two-sided t-test. All experiments were performed at least three times. Differences with ${ }^{\mathrm{P}}<0.005$ versus control group $(-)$, and $* \mathrm{P}<0.05$ and $* * \mathrm{P}<0.001$ versus TNF $\alpha+\mathrm{IFN} \gamma$ treatment group $(+)$ were considered statistically significant.

immune responses remains undeveloped, we recently have reported a specific role of SOD3 in leading immune response (19).

SOD3 is a secretory protein and is composed of two subunits. One is a full length SOD3, and the other is $209 \mathrm{E}$ which lacks HBD. HBD of SOD3 could be eliminated by intracellular proteolytic cleavage before secretion (20). When we treated rh209E to the cells, no signals of 209E signal were found in the cell lysate. However, full length rhSOD3 was found in the cell lysate in the presence of $\mathrm{Zn}^{2+}$, in a dose dependent manner. These results suggest that HBD of SOD3 played a role in the internalization of SOD3 under the presence of $\mathrm{Zn}^{2+}$; in particular, $\mathrm{Zn}^{2+}$ enhanced the internalization of SOD3 from the media to cell lysate. HBD of SOD3 is likely to chelate $\mathrm{Zn}^{2+}$ to permit interaction with the negatively charged oligosaccharides.

Interestingly, the expression of cytokines such as IL-1 $\beta$, IL-1 $\alpha$, TNF- $\alpha$, IL-8, and IL-6 decreased with rhSOD3 and rh209E. These recombinant proteins are effective enzymes which reduce the mRNA level of cytokines after treatment with TNF $\alpha$ and IFN $\gamma$ to HaCaT. However, rhSOD3 significantly reduces the inflammatory cytokines in the presence of $\mathrm{Zn}^{2+}$. The inhibitory effect of rhSOD3 on inflammatory cytokines is dependent on the presence of $\mathrm{Zn}^{2+}$ which elevates the internalization of rhSOD into the cell and translocation into the nuclear region. These results suggest that $\mathrm{Zn}^{2+}$ promotes anti-inflammatory effects of rhSOD3 by increasing cellular 
association via $\mathrm{HBD}$ of rhSOD3. In addition, $\mathrm{Zn}^{2+}$ elevates the delivery of HBD proteins into the skin, similar to SOD3. This property would be a useful enhancer in the delivery of small and large therapeutics having poor skin penetration, especially proteins possessing HBD. Optimization of the procedure will help in overcoming fundamental barriers in the development of HBD proteins, including rhSOD3, as a novel biomedicine.

\section{MATERIALS AND METHODS}

\section{Preparation of recombinant SOD3}

The recombinant SOD3 was prepared as described previously (21). 293T cells were transiently transfected with SOD3 construct for $48 \mathrm{~h}$. The supernatant was collected, and subjected to purification using a column containing Ni-NTA agarose (Qiagen), and dialysis. The purified SOD3 activity was measured with a SOD assay kit (Dojindo) as described previously (22). For injection in mice or treatment in vitro, SOD3 was filtered to eliminate endotoxins. SOD3 has two isoforms, the full length and the proteolytic variant 209E (which is devoid of HBD at the C-terminal end). The full length human SOD3 and proteolytic cleaved form 209E variant (from Met1 to Glu227) containing a C-terminal His6 tag, were inserted into pcDNA3.1 (Invitrogen) using HindlII and EcoRI or HindIII and Xbal, respectively. Plasmids encoding hSOD3 and 209E variants were transfected into 293T-EBNA cells with Attractene (Qiagen), based on the manufacturer's instructions. One day after transfection, the media was replaced with serum-free Dulbecco's Modified Eagle Medium (DMEM).

\section{Protein expression and purification}

Five days after transfection, culture media containing rhSOD3 were collected, filtered, and loaded onto HiTrap Chelating HPcolumn (GE Healthcare). After loading, the column was washed with more than 50 column volumes of washing buffer, $50 \mathrm{mM} \mathrm{NaPO}_{4}, 500 \mathrm{mM} \mathrm{NaCl}$, and $30 \mathrm{mM}$ imidazole. Next, the rhSOD3 and rh209E were eluted by increasing the elution buffer containing $500 \mathrm{mM}$ imidazole, followed by dialysis in PBS. The concentration of purified rhSOD3 was determined based on a bovine serum albumin standard curve, with a protein assay dye (Bio-Rad).

\section{Activity assay for SOD}

To measure the enzymatic activity of rhSOD3, the rate of superoxide radical formation was quantified by spectrophotometer. A $20 \mu \mathrm{l}$ sample was mixed with $200 \mu \mathrm{l}$ of $200 \mu \mathrm{M}$ xanthine (Sigma) and $50 \mu \mathrm{M}$ WST-1 (Dojindo) in PBS. After adding 0.0005 unit XOD (Sigma), the increase in the formazan dye was immediately recorded using a colorimetric method, at A450. The generation of a formazan dye was determined kinetically, and absolute SOD activity was determined from the dilution factor exhibiting $50 \%$ inhibition (IC50) on the inhibition curve. The purified SOD3 activity was measured with a SOD assay kit (Dojindo), as described previously (22).

\section{Western blot analysis}

Western blot was performed as previously described (23). Briefly, the cells were lysed with radio-immuno-precipitation assay buffer (2 mM EDTA, $137 \mathrm{mM} \mathrm{NaCl}, 20 \mathrm{mM}$ Tris- $\mathrm{HCl}$ (pH 8.0), $1 \mathrm{mM}$ sodium vanadate, $10 \mathrm{mM} \mathrm{NaF}, 1 \mathrm{mM}$ PMSF, $1 \%$ Triton X-100, 10\% glycerol, and a protease inhibitor cocktail) and harvested immediately. The samples were loaded onto sodium dodecyl sulfate-polyacrylamide gels for electrophoresis and subsequently transferred onto polyvinylidene fluoride membranes obtained from Millipore (Bedford, MA, USA). After membranes were blocked, they were incubated with specific primary antibodies overnight at $4^{\circ} \mathrm{C}$, with gentle agitation. The membranes were washed and incubated with a horseradish peroxidase-conjugated secondary antibody for $1 \mathrm{~h}$ at room temperature. Bands were detected using ECL Plus western blotting detection reagents from Amersham Biosciences Co (Piscataway, NJ, USA).

\section{Measurements of nucleus accumulation for rhSOD3}

In order to visualize the rhSOD3 and rh209E distribution within the cell, two kinds of the SOD3 proteins were labeled with FITC (Fluorescein isothiocyanate). Specifically, PBS phosphate buffered saline (PBS) was labeled by reaction for 2 $\mathrm{h}$ at room temperature with FITC-labeled rhSOD3 protein in 1 $\mathrm{mg}$ to $100 \mathrm{mg}$ of the buffer solution. rhSOD3 or rh209E were prepared with FITC-labeled protein solution, in order to remove the unreacted FITC by high-speed liquid chromatography devices for protein purification (FPLC, Fast Protein Purification Liquid Chromatography), and were then purified by desalting columns. HaCaT was cultured on $8 \mathrm{~mm}$ cover slip to a $1 \times 10^{4}$ cells/well in dispensing a rear, in IMDM (Isocove's modified Dulbecco's medium, using the GIBCO) medium supplemented with $10 \%$ FBS, 100 units $/ \mathrm{ml}$ penicillin, $100 \mathrm{~g} / \mathrm{ml}$ streptomycin and incubated under conditions of $5 \% \mathrm{CO}_{2}$, $37^{\circ} \mathrm{C}$. Once the cells attached to the coverslip and attained 30 to $40 \%$ confluency, the cells were starved in serum free media and cultured for $24 \mathrm{~h}$. At $50-60 \%$ confluency, the cells were treated with isolated and purified rhSOD3 or rh209E of $10 \mu \mathrm{g}$ protein labeled with FITC and Hoechst33342 fluorescence staining, under same culture conditions mentioned above. After the requisite incubation, the cells were viewed under confocal fluorescence microscopy (confocal laser scanning microscope, Carl Zeiss LSM 510).

\section{Anti-inflammatory effects of rhSOD3}

At $70 \%$ confluency, HaCaT were starved in serum-free DMEM for $6 \mathrm{~h}$ prior to treatment with $10 \mathrm{ng} / \mathrm{ml} \mathrm{TNF}-\alpha$ and $100 \mathrm{U} / \mathrm{ml}$ IFN- $\gamma$. Purified rhSOD3 and 209E were added to cells (200 $\mathrm{U} / \mathrm{mL}$ concentration), followed by treatment with various concentration of $\mathrm{ZnCl}_{2}(0-10 \mu \mathrm{M})$. Cells were harvested after $24 \mathrm{~h}$ incubation by directly adding SDS sample buffer containing protease inhibitors. p-STAT3, STAT3, GAPDH, and rhSOD3 
were analyzed by western blot analysis, using anti-p-STAT3, p-STAT3, GAPDH (Santa Cruz Biotechnology, CA, USA) and anti- hSOD3 (AbCam, Cambridge, UK) antibodies.

\section{RNA isolation and quantitative real-time PCR}

Total RNA was isolated from cells or tissue using an RNeasy Mini Kit (Qiagen, Valencia, CA, USA). Complementary DNA was synthesized using a QuantiTect Reverse Transcription Kit (Qiagen, Valencia, CA, USA). Quantitative real-time PCR was performed using the KAPA SYBR fast qPCR Kit (KAPA biosystems, Woburn, MA, USA), as previously described (24). The results were normalized to glyceraldehyde 3-phosphate dehydrogenase (GAPDH) gene expression. The PCR conditions were $1 \mathrm{cycle}$ at $95^{\circ} \mathrm{C}$ for $5 \mathrm{~min}$, followed by 35 cycles at $96^{\circ} \mathrm{C}$ for $20 \mathrm{~s}, 60^{\circ} \mathrm{C}$ for $20 \mathrm{~s}$ and $72^{\circ} \mathrm{C}$ for $20 \mathrm{~s}$, and ending with one cycle at $72^{\circ} \mathrm{C}$ for $5 \mathrm{~min}$. Primers used in this experiment were purchased from Qiagen.

\section{Histological analysis}

After shaving the mouse dorsal skin, $50 \mu \mathrm{l}$ of a mixture of rhSOD3 (2000 unit) and $\mathrm{ZnCl}_{2}(50 \mathrm{mM})$ was treated. Only rhSOD3 (2000 unit) was applied to the mice, and this group was designated as the rhSOD3 treatment group. After $1 \mathrm{~h}$ exposure, the skin was fixed with $4 \%$ paraformaldehyde in phosphate buffered saline for $24 \mathrm{~h}$, washed with tap-water, dehydrated with grade ethanol, and then embedded in paraffin. The paraffin blocks were cut in $4-\mu \mathrm{m}$ thick sections, mounted on glass slides, dewaxed, rehydrated with grade ethanol, and stained with FITC and Hoechst33342 fluorescence staining. Analysis was carried out using a fluorescence attached microscope (Olympus, Tokyo, Japan).

\section{Statistical analysis}

Data were presented as means \pm SEM, and statistical comparisons between groups were made with the unpaired twosided t-test. All experiments were performed at least three times.

\section{ACKNOWLEDGEMENTS}

This work was supported by the Industrial Technology Innovation program (10063322, Development of Animal Cell Culture based hEC-SOD for Management of Atopic Dermatitis) funded By the Ministry of Trade, industry \& Energy.

\section{CONFLICTS OF INTEREST}

The authors have no conflicting financial interests.

\section{REFERENCES}

1. Serra V, von Zglinicki T, Lorenz M and Saretzki G (2003) Extracellular superoxide dismutase is a major antioxidant in human fibroblasts and slows telomere shortening. J Biol Chem 278, 6824-6830

2. Teoh ML, Fitzgerald MP, Oberley LW and Domann FE
(2009) Overexpression of extracellular superoxide dismutase attenuates heparanase expression and inhibits breast carcinoma cell growth and invasion. Cancer Res 69, 6355-6363

3. Wheeler MD, Smutney OM and Samulski RJ (2003) Secretion of extracellular superoxide dismutase from muscle transduced with recombinant adenovirus inhibits the growth of B16 melanomas in mice. Mol Cancer Res 1, 871-881

4. Laukkanen MO, Kivelä A, Rissanen $\mathrm{T}$ et al (2002) Adenovirus-mediated extracellular superoxide dismutase gene therapy reduces neointima formation in balloondenuded rabbit aorta. Circulation 106, 1999-2003

5. Marklund SL (1990) Expression of extracellular superoxide dismutase by human cell lines. Biochem J 266, 213-219

6. Marklund SL (1992) Regulation by cytokines of extracellular superoxide dismutase and other superoxide dismutase isoenzymes in fibroblasts. J Biol Chem 267, 6696-6701

7. Stralin P and Marklund SL (2000) Multiple cytokines regulate the expression of extracellular superoxide dismutase in human vascular smooth muscle cells. Atherosclerosis 151, 433-441

8. Ookawara T, Kizaki T, Takayama E et al (2002) Nuclear translocation of extracellular superoxide dismutase. Biochem Biophys Res Commun 296, 54-61

9. Green $M$ and Loewenstein PM (1988) Autonomous functional domains of chemically synthesized human immunodeficiency virus tat trans-activator protein. Cell 55, 1179-1188

10. Frankel AD and Pabo CO (1988) Cellular uptake of the tat protein from human immunodeficiency virus. Cell 55, 1189-1193

11. Dixon JE, Osman G, Morris GE et al (2016) Highly efficient delivery of functional cargoes by the synergistic effect of GAG binding motifs and cell-penetrating peptides. Proc Natl Acad Sci U S A 113, E291-299

12. Shrestha P, Yun JH, Kim WT, Kim TY and Lee W (2016) Cloning, purification, and characterization of recombinant human extracellular superoxide dismutase in SF9 insect cells. Mol Cells 39, 242-249

13. Park KY, Kim EY, Lee W, Kim TY and Kim WT (2016) Expression, subcellular localization, and enzyme activity of a recombinant human extra-cellular superoxide dismutase in tobacco (Nicotiana benthamiana L.). Protein Expr Purif 119, 69-74

14. Ryu K, Kim YH, Kim Y, Lee JS, Jeon B and Kim TY (2008) Increased yield of high-purity and active tetrameric recombinant human EC-SOD by solid phase refolding. J Microbiol Biotechnol 18, 1648-1654

15. Auten RL, O'Reilly MA, Oury TD, Nozik-Grayck E and Whorton MH (2006) Transgenic extracellular superoxide dismutase protects postnatal alveolar epithelial proliferation and development during hyperoxia. Am J Physiol Lung Cell Mol Physiol 290, L32-40

16. Gongora MC, Lob HE, Landmesser $U$ et al (2008) Loss of extracellular superoxide dismutase leads to acute lung damage in the presence of ambient air: a potential mechanism underlying adult respiratory distress syndrome. Am J Pathol 173, 915-926

17. Juul K, Tybjaerg-Hansen A, Marklund S, Lange $P$ and 
Nordestgaard BG (2006) Genetically increased antioxidative protection and decreased chronic obstructive pulmonary disease. Am J Respir Crit Care Med 173, 858-864

18. Oury TD, Day BJ and Crapo JD (1996) Extracellular superoxide dismutase in vessels and airways of humans and baboons. Free Radic Biol Med 20, 957-965

19. Kwon MJ, Jeon YJ, Lee KY and Kim TY (2012) Superoxide dismutase 3 controls adaptive immune responses and contributes to the inhibition of ovalbumin-induced allergic airway inflammation in mice. Antioxid Redox Signal 17, 1376-1392

20. Kim S, Kim HY, Kim JH et al (2015) Enhancement of potency and stability of human extracellular superoxide dismutase. BMB Rep 48, 91-96

21. Kwon MJ, Han J, Kim BH, Lee YS and Kim TY (2012) Superoxide dismutase 3 suppresses hyaluronic acid fragments mediated skin inflammation by inhibition of toll-like receptor 4 signaling pathway: superoxide dismutase 3 inhibits reactive oxygen species-induced trafficking of toll-like receptor 4 to lipid rafts. Antioxid Redox Signal 16, 297-313

22. Jeon B, Kim BH, Lee YS, Kim S, Yoon JB and Kim TY (2011) Inactive extracellular superoxide dismutase disrupts secretion and function of active extracellular superoxide dismutase. BMB Rep 44, 40-45

23. Kim $Y$, Kim BH, Lee $H$ et al (2011) Regulation of skin inflammation and angiogenesis by EC-SOD via HIF-1a and NF-kB pathways. Free Radic Biol Med 51, 1985-1995

24. Uematsu T, Nagashima S, Umemura K, Kanamaru M and Nakashima M (1994) Pharmacokinetics and safety of intravenous recombinant human superoxide dismutase (NK341) in healthy subjects. Int J Clin Pharmacol Ther 32, 638-641 